Published in "Spectrochimica Acta Part B: Atomic Spectroscopy 136 (): 23-33, 2017" which should be cited to refer to this work.

\title{
High energy resolution off-resonant spectroscopy: A review
}

\author{
Wojciech Błachucki ${ }^{a}$, Joanna Hoszowska ${ }^{b}$, Jean-Claude Dousse ${ }^{b}$, Yves Kayser ${ }^{c}$, Regina Stachura ${ }^{d}$, \\ Krzysztof Tyrała $^{\mathrm{d}}$, Klaudia Wojtaszek ${ }^{\mathrm{d}}$, Jacinto Sáa, e,*, Jakub Szlachetko ${ }^{\mathrm{a}, \mathrm{d}, *}$ \\ a Institute of Physical Chemistry, Polish Academy of Sciences, Warsaw 01-224, Poland \\ ${ }^{\mathrm{b}}$ Department of Physics, University of Fribourg, Chemin du Musée 3, CH-1700 Fribourg, Switzerland \\ ' Physikalisch-Technische Bundesanstalt (PTB), Abbestr. 2-12, Berlin 10587,Germany \\ d Institute of Physics, Jan Kochanowski University, Kielce 25-406, Poland \\ e Department of Chemistry, Uppsala University, Uppsala 752 37, Sweden
}

\begin{abstract}
We review the high energy resolution off-resonant spectroscopy (HEROS) technique. HEROS probes the unoccupied electronic states of matter in a single-shot manner thanks to the combination of off-resonant excitation around atomic core states using wavelength dispersive X-ray detection setups. In this review we provide a general introduction to the field of X-ray spectroscopy together with the specification of the available X-ray techniques and X-ray methodologies. Next, the theoretical description of the HEROS approach is introduced with a special focus on the derivation of the X-ray emission and X-ray absorption correspondence relation at off-resonant excitation conditions. Finally, a number of experimental HEROS reports are reviewed in the field of chemistry and material science. We emphasize the applicability of HEROS to pulsed $\mathrm{X}$-ray sources, like X-ray free electron lasers, and support the review with experimental examples. The review is complemented with perspectives on and possible further applications of the HEROS technique to the field of X-ray science.
\end{abstract}

\section{Contents}

1. Introduction

2. Theoretical background . . . . . . . . . . . . . . . . . . . . . . . . . . . . . . . . . . . . . . . . . . . . . . . . 25

2.1. Introduction $\ldots \ldots \ldots \ldots \ldots$



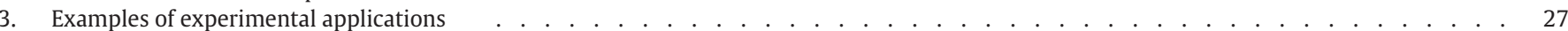

3.1. HEROS and the self-absorption effect ． . . . . . . . . . . . . . . . . . . . . . . . . . . . . . . . . . . . . . . . . 27

3.2. HEROS for time-resolved chemical speciation . . . . . . . . . . . . . . . . . . . . . . . . . . . . . . . . . 27

3.2.1. In situ study on $\mathrm{Pt}(\mathrm{acac})_{2}$ decomposition ～. . . . . . . . . . . . . . . . . . . . . . . . . . . . . . . . . . . . . 27

3.2.2. In situ study on $\mathrm{Pt}^{\prime} / \mathrm{Al}_{2} \mathrm{O}_{3}$ oxidation-reduction cycles $\quad \ldots \ldots \ldots$

abbreviations: XAS, X-ray absorption spectroscopy; XANES, X-ray absorption near-edge structure (also called Near-edge X-ray absorption fine structure, NEXAFS); EXAFS, Extended X-ray absorption fine structure; EY, Electron yield; XES, X-ray emission spectroscopy (also called Second order X-ray spectroscopy or Photon-in/photon-out X-ray spectroscopy); NXES, Non-resonant XES or Normal XES, XRF - X-ray fluorescence, RIXS - Resonant inelastic X-ray scattering (also called Resonant X-ray Raman scattering); REXS, Resonant elastic X-ray scattering; TFY, Total fluorescence yield; PFY, Partial fluorescence yield; HR-XAS, High-resolution XAS (also called High-energy resolution fluorescence detected XAS, HERFD-XAS); HEROS, High-energy resolution off-resonant spectroscopy; LBS-XANES, Lifetime-broadening-suppressed XANES (also called Lifetime-broadeningremoved XANES, LBR-XANES); LBF-XANES, Lifetime-broadening-free XANES.

* Corresponding authors.

E-mail addresses: jacinto.sa@kemi.uu.se (J. Sa), jakub.szlachetko@ujk.edu.pl (J. Szlachetko). 


\section{Introduction}

The structure and functioning of matter is currently widely studied by means of different spectroscopy disciplines. One of them, $\mathrm{X}$-ray spectroscopy, is focused on examining the energy distribution of inner- and outer-shell atomic electrons quantum states in different conditions [1]. The study of the interaction of X-ray radiation with atoms allows determining the electronic structure of matter as well as investigating the intra/inter-atomic and intra/inter-molecular electronic processes. X-ray spectroscopy is extensively used for elemental identification [2], determination of the atomic data [3,4], plasma diagnostics [5] and chemical analysis. Hence this technique found applications in many research fields like physics, chemistry, medicine, biology and material sciences (see, e.g., Refs. [6-12]).

$\mathrm{X}$-ray radiation can interact with matter through photoabsorption or X-ray scattering. The interaction of an X-ray photon with atomic electrons may induce different atomic decay processes which can lead to the emission of electromagnetic radiation. These processes dictate the subdivision of X-ray spectroscopy into different methodologies as shown in Fig. 1. The measurement and the analysis of the energy distribution of the X-ray radiation emitted in these processes (i.e., emission spectrum) are the scope of the discipline called X-ray emission spectroscopy (XES) $[13,14]$. In non-resonant XES (NXES), atoms are irradiated with X-rays having an energy largely above a given atomic energy level. The interaction with the excitation beam produces vacancies in the atomic energy levels with a lower binding energy and leaves the atoms in an excited state. The atoms' ground state is re-established through a fluorescence - cascade of spontaneous electron transitions between energy levels, each transition leading possibly to the emission of an X-ray photon. In NXES studies the energy of the incident photons does not need to be well defined as long as it remains above the studied ionization threshold. In case the incident photon energy is close to a given atomic energy level X-ray emission has unique properties and XES applied is then called Resonant XES (RXES). Resonant X-ray emission can occur via fluorescence, like NXES, or via a coherent absorption-emission process called Resonant inelastic X-ray scattering (RIXS). The energy of the fluorescence photons is strictly correlated with the atomic energy levels taking part in the electron transitions which may occur between two core levels (core-to-core level transition) or between a valence level and a core level (valence-to-core level transition). The emission spectra recorded for valence-to-core level transitions provide information on the occupancy of the valence orbitals. NXES is thus an element-specific tool allowing determining not only the energy differences between the atomic levels but also of the density of occupied electronic states. Valence-to-core electron transitions are of interest because valence levels are particularly sensitive to the spatial distribution of the surrounding atoms. Depending on the chemical environment of the atoms from which the fluorescence photons are measured, the measured valence-to-core X-ray emission lines may have different intensities and energies. NXES is therefore used in the study of the chemical environment of the fluorescing atoms, in particular the ligand orbitals and bond distances (see, e.g., Refs. [15-17]).

The dependence of X-ray absorption in the studied material on the incident photon energy is studied in X-ray absorption spectroscopy (XAS) $[18,19]$. The main XAS methods are: electron yield, transmission mode, fluorescence mode (or fluorescence yield) [20,21]. In electron yield (EY) XAS studies, the studied sample is irradiated with an X-ray beam and the electric current induced in the sample is measured as a function of the incident photon energy. Indeed the interaction with radiation leads to an increase of the number of free electrons in the material, mainly because of the photoelectrons and Auger electrons ejected from the atoms as a result of photoabsorption. The electron yield can be therefore used as a measure of the photoabsorption coefficient and it has been shown that it provides the same result as compared to other XAS methods [21,22]. In the transmission mode the X-ray absorption spectrum is derived from the X-ray beam intensities incident on the sample and transmitted through the sample using the Bouguer-Lambert-Beer law. Fluorescence mode XAS makes use of XES methods and is focused on the study of how the fluorescence intensity changes with the incident photon energy. It has been observed that the fluorescence yield varies with the photoabsorption coefficient and its dependence on the incident beam energy provides the same information as the transmission mode absorption spectra XAS [20]. The XAS spectrum can be obtained in the fluorescence mode using either the total fluorescence yield (TFY) or the partial fluorescence yield (PFY). TFY is the fluorescence yield integrated over a broad emission energy range, while for the PFY the integration is done over a selected emission energy range. In the particular case of PFY, where the fluorescence yield is integrated over an energy band centered on a given fluorescence line and narrower than the natural linewidth of the latter, fluorescence mode XAS is referred to as high resolution XAS (HR-XAS) or highenergy resolution fluorescence detected XAS (HERFD-XAS) [23,24]. Absorption spectra measured in HR-XAS reveal more detailed information than the ones obtained in transmission mode studies and are extensively used in chemical speciation (see, e.g., Refs. [25-28]). In HR-XAS measurements typically the core-to-core X-ray emission lines are chosen. They are characterized by a much higher intensity as compared to the fluorescence originating from valence-to-core electronic transitions and by a much better energy separation.

While X-ray absorption spectroscopy carries information on the energy distribution of the unoccupied electronic states (density of unoccupied states) in matter, X-ray emission spectra, on the other hand, provide data on the density of occupied states. In off-resonant conditions, i.e. when an incident beam with a well defined photon energy fixed below a given ionization threshold is used, the X-ray emission spectra present in addition a strong dependence on the density of unoccupied states. The first measurements under off-resonant conditions were performed by Sparks [29], who did a systematic research on off-resonant X-ray emission from different elements. The experimental setup he used, an X-ray tube with a single crystal monochromator and an energy-dispersive solid-state detector, was of sufficient resolving power to allow for the observation of asymmetric structures in the measured off-resonant XES spectra. Moreover, the position of the features in the observed spectral distribution depended on the energy of the atomic state from which the radiative decay occurred. Few years after Sparks' pioneering experiment, Tulkki and Åberg, starting from the generalized Kramers-Heisenberg formula $[30,31]$, derived a formula describing the cross section for the inelastic X-ray scattering process in the off-resonant regime. Their discovery shed new light to the interpretation of off-resonant XES spectra revealing a dependence of the off-resonant spectral features' shape on the density of unoccupied electronic states of the interacting atoms. This dependence could not be observed in the experiment of Sparks because of the low energy resolution of the used detection 


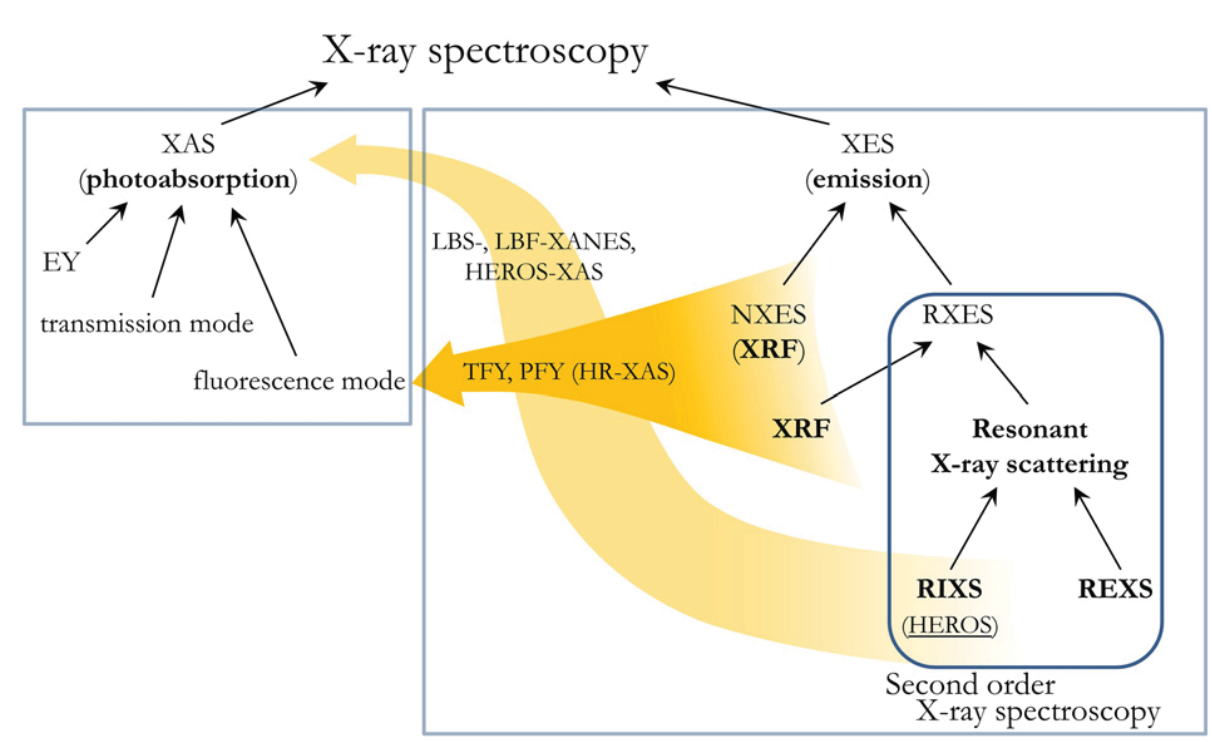

Fig. 1. Outline of X-ray spectroscopy methodologies. The terms in bold refer to physical processes, the remaining ones to X-ray spectroscopy methods. The meaning of the abbreviations is presented at the bottom of the title page. The emission spectra of photons scattered in the RIXS process reflect the density of unoccupied states. The resonant emission spectra, however, contain contribution of photons from both RIXS and much stronger XRF. In the HEROS approach, where only the RIXS photons are targeted, the incident beam energy is fixed below the absorption edge of interest - low enough to not induce normal fluorescence but possibly high enough to maintain sufficient scattering cross section. Thus, the incident beam energy is fixed at the value of typically 2-5 times the core level's lifetime broadening below the core level's binding energy which is defined by the authors as the upper limit of the off-resonant regime.

setup. Because inelastic X-ray scattering is characterized by a low cross section in the off-resonant regime, only few off-resonant XES studies were successfully accomplished since the work of Tulkki and Åberg. Nonetheless, with the development of wavelength-dispersive spectrometry (i.e. crystal X-ray spectrometers) the modulation of the off-resonant XES spectral structures' shape by the density of unoccupied states was confirmed experimentally with both conventional $\mathrm{Cu}$ $K \alpha \mathrm{X}$-ray sources [32,33] and synchrotron radiation sources [34-37].

The major problems of off-resonant XES studies on the density of unoccupied states are the very low cross section of the inelastic Xray scattering ( $\sim 10^{3}$ smaller than the photoabsorption cross section) and the need of high-energy resolution detection systems. To overcome them, single- or multiple-crystal spectrometers operating in a point-to-point geometry are typically used at synchrotrons and XFEL facilities [34,35,38,39]. These detection systems, however, allow only for point-by-point measurements of the emission spectra which, because of definitely extended recording time for the full spectra, makes them inapplicable in many studies, especially at XFELs. Only recently, a wavelength-dispersive von Hamos-geometry-based spectrometer was used to measure in high resolution single-shot spectra of off-resonantly scattered X-rays, establishing thus the high energy resolution off-resonant spectroscopy (HEROS) [40-42]. Until now different research studies on HEROS have been realized at synchrotrons as well as at XFELs and the method found numerous applications in in situ time-resolved chemical speciation experiments [40,41,43-51]. HEROS is a new approach and is becoming more and more recognized in the field of X-ray spectroscopy (see, e.g., Refs. [42,52-64]).

\section{Theoretical background}

\subsection{Introduction}

X-ray emission spectroscopy (XES) represents a number of experimental methods dedicated to the measurements of spectra of X-ray radiation emitted from matter. Different features of electronic structure of atoms may be probed by means of off-resonant, resonant and non-resonant XES in gases, liquids and solids. RXES corresponds to XES applied in resonant conditions, i.e., when the incident photon energy is close-to or around the energy threshold of an atomic core state. In principle, RXES combines the aspects of simultaneous probing of occupied and unoccupied electronic states in a single experimental approach. In general two X-ray scattering processes may be distinguished for resonant X-ray-matter interaction: Resonant elastic X-ray scattering (REXS) $[65,66]$ and Resonant inelastic X-ray scattering (RIXS) $[67,68]$. The REXS process delivers information on the spatial modulations of charge, spin and orbital degrees of freedom, whereas the RIXS process provides data on the density of unoccupied electronic states and the geometric orientation of the interacting atoms. The high energy resolution off-resonant spectroscopy, in this respect may be regarded as complementary since it aims at measurements of the inelastic scattering of X-rays under off-resonant conditions, i.e., on the inelastic scattering of photons having energies below the ionization threshold.

\subsection{Theoretical description}

For incident photon energies being tuned around the binding energy of an atomic core state an enhanced X-ray scattering signal is observed. The X-ray radiation emitted in inelastic scattering has unique properties and its spectral energy distribution carries information on the electronic structure of the scattering atom.

Fig. 2 presents a schematic drawing of the resonant inelastic X-ray scattering process on core atomic states. The scattering cross sections vary depending on whether the scattering process involves discrete or continuum states. At incident X-ray energies that are tuned below the ionization threshold, i.e., at off-resonant conditions, the excitation and transition decay paths should be regarded as a coherent process where the additional energy of the photoelectron is provided at the expense of the emitted photon energy. The intensity and energy of the emission signal induced at off-resonant excitations provide information on the unoccupied states thanks to the energy conservation for the scattering process, i.e., $\hbar \omega_{2}(E)=\hbar \omega_{1}-\left|E_{f}\right|-E$. We would like to stress, that unlike in resonant or non-resonant XES, the energy of the emitted X-rays at off-resonant scattering conditions is given by the energy of the incident $\mathrm{X}$-rays and by the final state energy $\left(\hbar \omega_{2}(E) \leqslant \hbar \omega_{1}-\left|E_{f}\right|\right)$.

Because of the energy detuning from the core-hole energy and the resulting coherent nature of the scattering process, only the final state lifetime contributes to the broadening of the off-resonant X-ray emission spectra. For photon excitation energies $\hbar \omega_{1}$ corresponding to an absorption threshold $\left|E_{i}\right|$, i.e., on-resonance, the X-ray scattering 



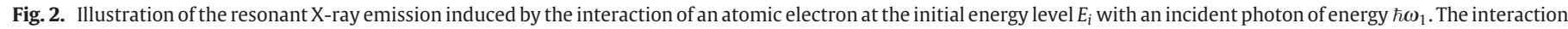

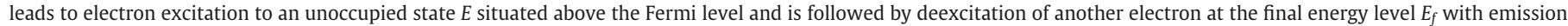

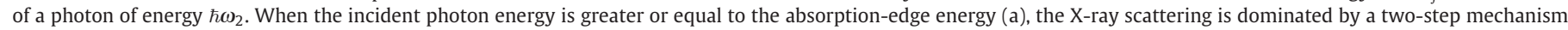

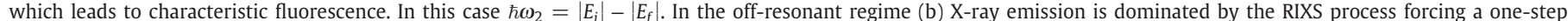

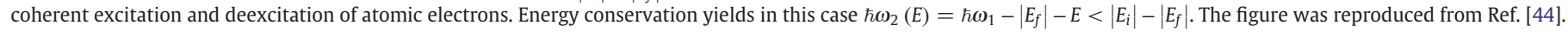

leads to resonance fluorescence. For incident photon energies above a given absorption threshold an electron from a core level (initial) is excited to an unoccupied state of energy $E$ localized in the continuum (see Fig. 2 (a)). The excitation is followed by the decay of an electron from another core level (final) of energy $\left|E_{f}\right|$ and possibly the emission of X-ray fluorescence. Due to the energy conservation the emitted photon has an energy $\hbar \omega_{2}=\left|E_{i}\right|-\left|E_{f}\right|$ and the resulting emission spectrum is broadened by the lifetimes of initial and final states. For discrete atomic states with energy $E_{\text {discrete, }}$ the inelastic X-ray scattering follows the energy conservation with $\hbar \omega_{2}=\hbar \omega_{1}-\left|E_{f}\right|+$ $\left|E_{\text {discrete }}\right|$. When the incident energy is equal to the initial state-todiscrete state resonance, the photoelectron energy $E=\hbar \omega_{1}-\left|E_{i}\right|$ is equal to $E_{\text {discrete. }}$ In case of inelastic X-ray scattering on discrete states, the energy transfer (energy loss) $\hbar \omega_{1}-\hbar \omega_{2}$ is constant.

Based on the correspondence principle and by transforming the classical dispersion formula introduced by Lorentz, Kramers and Heisenberg derived equations describing the cross section for X-ray scattering [31]. The same result may be derived based on quantum electrodynamics and starting from Schrödinger equation as demonstrated by Dirac [69].

On the basis of the Kramers-Heisenberg model, Tulkki and Åberg developed formulas for differential cross sections for X-ray interaction with matter. These equations are suitable for a full description of resonant, non-resonant and off-resonant scattering processes in matter. Tulkki and Åberg considered off-resonant X-ray scattering $\left(\hbar \omega_{1} \ll\left|E_{i}\right|-\Gamma_{i}\right)$ within the dipole approximation $(\exp (\boldsymbol{l} \boldsymbol{k} \cdot \boldsymbol{r}) \approx 1)$. In this picture, the off-resonant scattering process may be described as an excitation of the initial core electron into an intermediate virtual state. This state decays then by a radiative transition from the higher core or valence state into the core-hole state. The schematic of the process is drawn in Fig. 3.

As reported by Åberg and Tulkki, the anisotropic interference term for the resonant and non-resonant paths is negligible, and thus the off-resonant X-ray scattering differential cross sections can be written as follows:

$$
\begin{aligned}
\frac{\mathrm{d} \sigma\left(\hbar \omega_{1}\right)}{\mathrm{d}\left(\hbar \omega_{2}\right)} \sim & \int_{0}^{\infty}\left[\frac{\left(\left|E_{i}\right|-\left|E_{f}\right|\right)\left(\left|E_{i}\right|+E\right)}{\left(\left|E_{i}\right|+E-\hbar \omega_{1}\right)^{2}+\Gamma_{i}^{2} / 4}\right. \\
& \times \frac{\Gamma_{f} / 2 \pi}{\left(\hbar \omega_{1}-\hbar \omega_{2}-\left|E_{f}\right|-E\right)^{2}+\Gamma_{f}^{2} / 4} \\
& \left.\times \frac{\hbar \omega_{2}}{\hbar \omega_{1}} g_{f \leftrightarrow i} \frac{\mathrm{d} g_{i}}{\mathrm{~d} E}(E)\right] \mathrm{d} E,
\end{aligned}
$$

where the energies of incoming and outgoing photons are denoted as $\hbar \omega_{1}$ and $\hbar \omega_{2}, E$ stands for the energy above the Fermi level of the excited electron. The oscillator strength for the final to initial state transition is represented by $g_{f \leftrightarrow i}$. The $\frac{d g_{i}}{d E}(E)$ represents the oscillator strength distribution for core shell absorption, and expressed as a function of the energy of the excited electron. The second term in Eq. (1) represents the normalized final state density function that accounts for the final state broadening $\Gamma_{f}$. This term also ensures the beforehand mentioned energy conservation $\hbar \omega_{2}(E)=\hbar \omega_{1}-\left|E_{f}\right|-E$.

The cross section equation may be further simplified by exchanging the Lorentz final state broadening with the Dirac delta function $\delta\left(\hbar \omega_{1}-\hbar \omega_{2}-\left|E_{f}\right|-E\right)$ that still ensures energy conservation for the scattering process. Thanks to this, the differential cross sections for off-resonant scattering may be written explicitly in an analytical form:

$$
\begin{aligned}
& \frac{\mathrm{d} \sigma\left(\hbar \omega_{1}\right)}{\mathrm{d}\left(\hbar \omega_{2}\right)} \sim \int_{0}^{\infty}\left[\frac{\left(\left|E_{i}\right|-\left|E_{f}\right|\right)\left(\left|E_{i}\right|+E\right)}{\left(\left|E_{i}\right|+E-\hbar \omega_{1}\right)^{2}+\Gamma_{i}^{2} / 4}\right. \\
& \times \delta\left(\hbar \omega_{1}-\hbar \omega_{2}-\left|E_{f}\right|-E\right) \\
& \left.\times \frac{\hbar \omega_{2}}{\hbar \omega_{1}} g_{f \leftrightarrow i} \frac{\mathrm{d} g_{i}}{\mathrm{~d} E}(E)\right] \mathrm{d} E \\
& \sim \frac{\left(\left|E_{i}\right|-\left|E_{f}\right|\right)\left(\left|E_{i}\right|-\left|E_{f}\right|+\hbar \omega_{1}-\hbar \omega_{2}\right)}{\left(\left|E_{i}\right|-\left|E_{f}\right|-\hbar \omega_{2}\right)^{2}+\Gamma_{i}^{2} / 4} \\
& \times \frac{\hbar \omega_{2}}{\hbar \omega_{1}} g_{f \leftrightarrow i} \frac{\mathrm{d} g_{i}}{\mathrm{~d} E}(E) \text {. }
\end{aligned}
$$

This equation provides the basis for high energy resolution offresonant spectroscopy. It connects directly X-ray absorption and X-ray emission signals through $\frac{\mathrm{d} \sigma\left(\hbar \omega_{1}\right)}{\mathrm{d}\left(\hbar \omega_{2}\right)}$ and $\frac{\mathrm{d} g_{i}}{\mathrm{~d} E}(E)$ terms. From the above equation the $\mathrm{X}$-ray absorption signal may be thus defined as:

$$
\begin{aligned}
\mathrm{XAS}=\frac{\mathrm{d} g_{i}}{\mathrm{~d} E}(E)= & \frac{1}{\mathrm{~A}} \frac{\mathrm{d} \sigma\left(\hbar \omega_{1}\right)}{\mathrm{d}\left(\hbar \omega_{2}\right)} \frac{1}{g_{f \leftrightarrow i}} \frac{\hbar \omega_{1}}{\hbar \omega_{2}} \\
& \times \frac{\left(\left|E_{i}\right|-\left|E_{f}\right|-\hbar \omega_{2}\right)^{2}+\Gamma_{i}^{2} / 4}{\left(\left|E_{i}\right|-\left|E_{f}\right|\right)\left(\left|E_{i}\right|-\left|E_{f}\right|+\hbar \omega_{1}-\hbar \omega_{2}\right)} .
\end{aligned}
$$

As the experimental HEROS yields at the energy $\hbar \omega_{2}$ are proportional to the differential cross section $\frac{d \sigma\left(\hbar \omega_{1}\right)}{d\left(\hbar \omega_{2}\right)}$ the latter can be replaced in Eq. (3) by the measured off-resonant intensity. For a given beam energy $\hbar \omega_{2}$ tuned below the core hole ionization threshold, the profile of the distribution $\frac{\mathrm{d} g_{i}}{\mathrm{~d} E}$ can thus be derived with this 


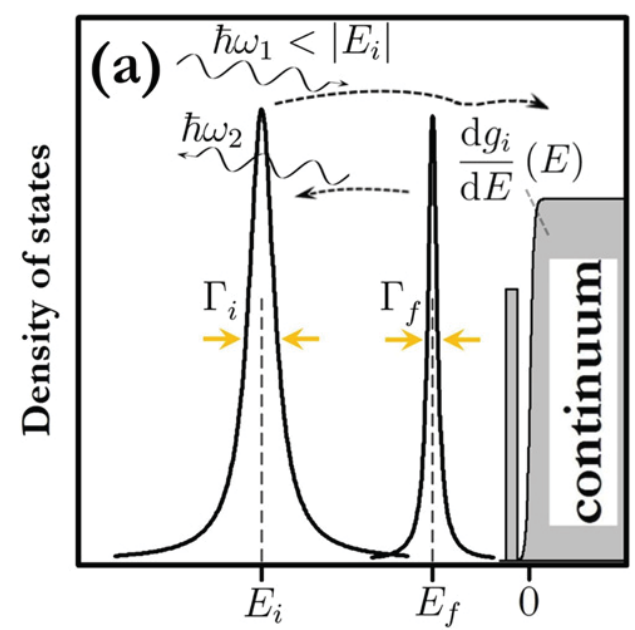

Energy

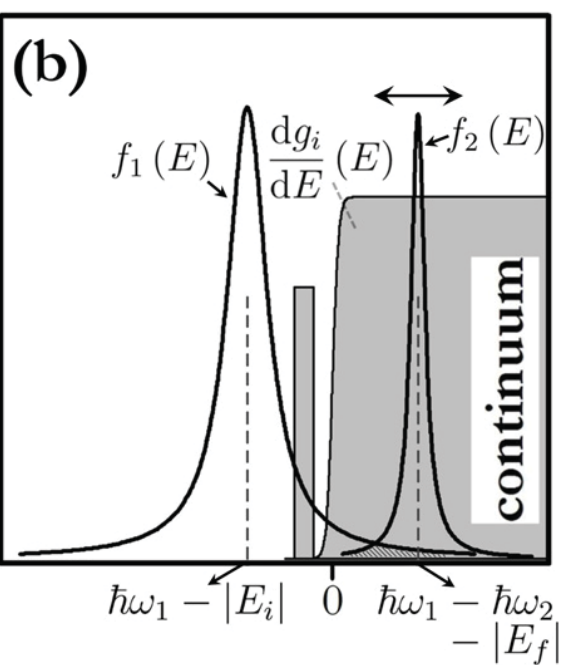

Energy



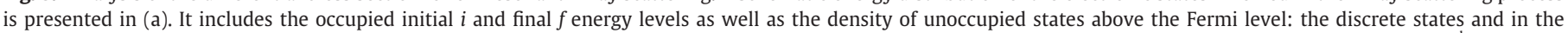

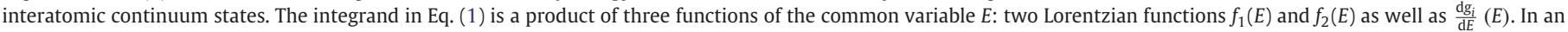

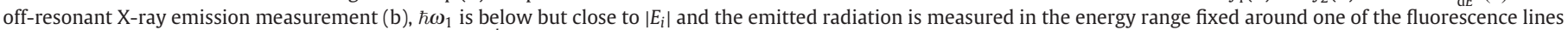



method, like in absorption measurements, from the corresponding HEROS spectra for a wide energy range $E$.

In general the shape of the HEROS profiles are characterized by a long low energy tail which is due to the Lorentzian shape of the involved initial atomic level. At the low energy limit $\left(\hbar \omega_{2} \rightarrow 0\right)$ the differential cross section $\frac{\mathrm{d} \sigma\left(\hbar \omega_{1}\right)}{\mathrm{d}\left(\hbar \omega_{2}\right)}$ vanishes. On the high energy side the sharp high-energy cutoff is defined by the energy conservation rule $\left(\hbar \omega_{2} \rightarrow 0\right)$. This high energy cutoff will move toward lower/higher energies when the energy of the incoming photons decreases or increases.

\section{Examples of experimental applications}

\subsection{HEROS and the self-absorption effect}

It has been known for many years that conventional total fluorescence mode XAS measurements are affected by the so-called self-absorption mechanism for concentrated samples [70]. This effect results from fundamental processes behind X-ray interaction with matter and thus cannot simply be avoided or omitted. From the application point of view of XAS based methodology in a wide range of disciplines, many approaches were proposed to circumvent this effect or account for it in the experimental data. In many scientific areas, however, self-absorption cannot be omitted because of the nature of the sample which cannot be diluted or thinned to the requested thickness. In this respect, the research on self-absorption effects is a science in itself.

In recent studies, the Ta $L_{3}$ absorption edge [44] was investigated by means of fluorescence and transmission mode XAS as well as by means of HEROS. As expected the absorption spectra measured in the fluorescence mode exhibit a strong self-absorption effect which causes a reduction of the detected spectral structures, both in the $\mathrm{X}$-ray absorption near edge structure (XANES) and in the extended $\mathrm{X}$-ray absorption fine structure (EXAFS) regions. It is important to note, that the main effect of the self-absorption mechanism arises from the sudden change of the photoabsorption coefficient when scanning the incident X-ray energy around an ionization threshold of an atom. This is not the case, however, for the HEROS technique, where both the incident and the emission X-ray energies are fixed during the acquisition. As a consequence, the probability of self-absorption of X-ray events is almost constant over the range of emission X-ray energies and thus the measured HEROS shape should be independent of the sample concentration or thickness that is, free of self-absorption effects.

The presence of self-absorption in the spectra measured by means of HEROS was investigated with different Ta metallic foils of nominal thicknesses in the range of few tens of microns. Since the HEROS spectrum contains information on the density of unoccupied states it can be converted to an absorption spectrum by means of the formula introduced by Kramers and Heisenberg [31] and modified by Tulkki and Åberg [30]. In this way, the conventionally measured spectra recorded in fluorescence and transmission modes by scanning the incident X-ray energy can be compared to a high degree of detail with the HEROS measurements. Indeed, the measured Ta $L \alpha_{1}$ HEROS emission spectra and the reconstructed Ta $L_{3}$ HEROSXAS absorption spectra were found to be independent of the target thickness and thus free of the self-absorption effect. This effect is presented in Fig. 4, where X-ray absorption spectra measured in fluorescence mode for different sample thicknesses are compared to reconstructed HEROS-XAS results.

The absence of modulation of the spectral shape by the selfabsorption mechanism allows investigation of strongly absorbing samples, making thus HEROS an alternative and complementary method to conventional XAS measurements. HEROS may thus be foreseen as an additional and complementary tool when precise information about specific absorption features, especially the socalled white lines and pre-edge peaks, and their strengths is crucial for chemical speciation or theoretical evaluation. The method may also be used as a test-measure to evaluate different experimental and methodological approaches aiming at precise corrections to the self-absorption effects in XAS.

\subsection{HEROS for time-resolved chemical speciation}

\subsubsection{In situ study on $\mathrm{Pt}(\text { acac })_{2}$ decomposition}

In the first HEROS application, the platinum(II) acetylacetonate complex $\mathrm{Pt}(\mathrm{acac})_{2}$ was studied in situ in real time throughout its decomposition in hydrogen [40]. $\beta$-diketonate metal complexes find various uses, e.g., as catalysts or addition to fuel or metal precursors 

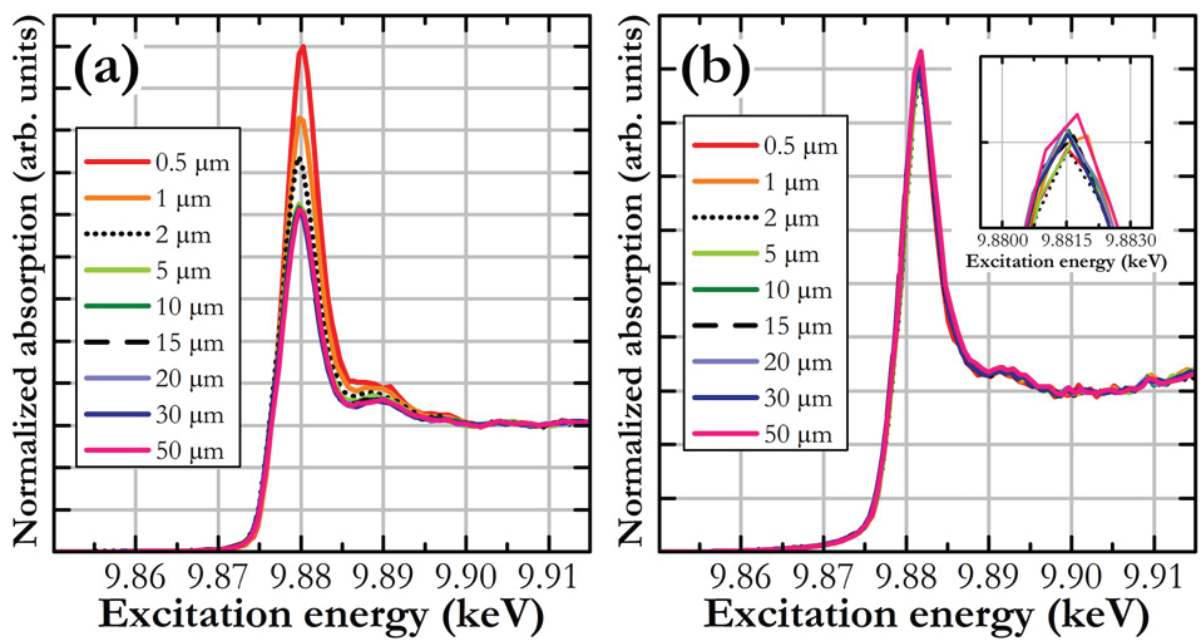

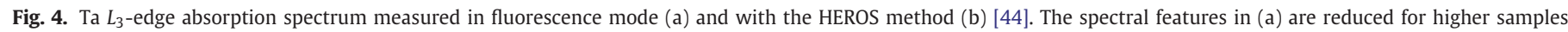
thicknesses while the sample thickness has no effect on the HEROS-XAS spectra. The figure was reproduced from Ref. [44].

and are attractive in industry, e.g., for chemical vapor deposition of superconductive layers and chemical vapor nucleation of metallic nanoparticles. Normally, the full decomposition of these metal complexes needs high temperature. This can, however, be overcome by adding to the system hydrogen or water. The decomposition processes are readily studied with X-ray absorption spectroscopy due to its chemical sensitivity and very high penetration depth allowing probing the system in situ. In XAS, however, the incident X-ray beam energy is scanned throughout an absorption edge of interest which limits the time resolution to as much as a few minutes. To observe a dynamic decomposition process that takes place within seconds, one needs a chemically sensitive spectroscopic method providing at the same time a high time resolution and high sensitivity through the high energy resolution.

In this work, HEROS was used to follow the Pt(acac) $)_{2}$ complex decomposition at a sub-second time resolution at a synchrotron. The sample was exposed to $\mathrm{H}_{2}$ and heated up to the temperature of $150{ }^{\circ} \mathrm{C}$ by flash heating. Pt $L \alpha_{1}$ HEROS spectra were recorded one by one with the acquisition time of $500 \mathrm{~ms}$ at the incident beam energy of $11.537 \mathrm{keV}$, i.e. $27 \mathrm{eV}$ below the Pt $L_{3}$ edge binding energy by means of von Hamos geometry-based spectrometer consisting of a cylindrically bent $\mathrm{Ge}(660)$ crystal and a micro-strip detector. The observed spectral structures' intensity and energy position evolved during the complex decomposition revealing a change of the density of unoccupied Pt $5 d$ states indicating the Pt atoms' oxidation state change. While the spectra measured at the beginning of the experiment were typical for $\mathrm{Pt}(\mathrm{acac})_{2}$, the final spectra were characteristic for metallic platinum particles. Using high-resolution transmission electron microscopy, the formed metallic particles were found to be about $4-8 \mathrm{~nm}$ big and highly crystalline. Spectral analysis also allowed a clear observation of Pt intermediate state in which the acac's decomposition residues covered only part of the Pt atoms causing their reduction and the subsequent formation of platinum nanoparticles. The experimental HEROS spectra recorded during Ptcomplex decomposition are plotted in Fig. 5. Analysis of white line intensity and energy edge position, shown in Fig. 5 (b), clearly indicates intermediate Pt-species in region marked as $\mathrm{B}$. The observed intermediate step during $\mathrm{Pt}(\mathrm{acac})_{2}$ decomposition in hydrogen was not reported in previous works [71].

\subsubsection{In situ study on $\mathrm{Pt} / \mathrm{Al}_{2} \mathrm{O}_{3}$ oxidation-reduction cycles}

The aspect of high energy resolution and availability of timeresolved studies offered by the HEROS technique was exploited in reduction and oxidation studies of $\mathrm{Pt} / \mathrm{Al}_{2} \mathrm{O}_{3}$ catalyst [43]. These studies were focused on the observation of electronic structure changes in catalytic system in real time. Platinum-based catalysts are the most important catalyst for oxidation and reduction reactions used by scientists and chemical industry. It is widely used for environmental protection, petroleum industry and chemical processes. Furthermore, platinum catalysts are the most important part of proton exchange membrane fuel cells (PEMFC) that are foreseen to replace the alkaline fuel cell. Platinum-based catalysts were shown to be very applicable in electrocatalytic processes, therefore a lot of work has been done in the field to understand its physical and chemical mechanisms.

In this field HEROS presents the potential for characterisation measurements with elemental specificity and to deliver detailed information on the density of unoccupied states. Thanks to the scanning free approach, the HEROS spectra can be registered with very high time resolution and an energy resolution independent of the width of the initial atomic core-state. During the experiment, in situ time-resolved HEROS spectra of $\mathrm{Pt} / \mathrm{Al}_{2} \mathrm{O}_{3}$ were acquired continuously around the Pt $L_{3}$ absorption edge and by detection of the Pt $L \alpha_{1}$ $\mathrm{X}$-ray radiation with a von-Hamos type spectrometer. The acquisition time per spectrum was $500 \mathrm{~ms}$ and the reduction and oxidation processes were monitored at $300{ }^{\circ} \mathrm{C}$. During this study, in order to increase the signal-to-noise ratio, about 120 loops were averaged, that suits to about $2 \mathrm{~h}$ total acquisition time. Gas-switches, allowing for controlling the reduction and oxidation cycles, were realized using fast-valves with $4 \% \mathrm{O}_{2}$ and $4 \% \mathrm{CO}$ gas feeds. The experimental data showed that during the reduction and oxidation steps the white line intensity and white line maximum energy position have changed, indicating a modification of the Pt $5 d$ density of empty states as the catalyst moves from oxide to metal and vice versa (see Fig. 6). The HEROS signal development during oxidation/reduction switches allowed to draw several conclusions. On the one hand, the reduction and oxidation final states are reached within $5 \mathrm{~s}$. The final state in case of the reduction process is reached faster than in the case of oxidation. Also during the reduction process the intermediate states are not registered because only a continuous decrease of energies and intensities of the white line is detected. On the other hand, for the oxidation mechanisms an additional intense peak is observed which suggests a metastable intermediate state. Indeed, thanks to HEROS's sensitivity and temporal resolution of the experiment the intermediate state during Pt-oxidation was detected and identified. Moreover, it is reported that the shift and the intensity changes as detected in HEROS spectra are not typical of a fully Pt-oxidized structure. In fact the spectra consist of a partly oxidized surface and a small amount of metallic platinum occurring in the core of the platinum nanoparticle. In summary, the studies allowed to conclude that the oxidation process is composed of two characteristic stages. The first step is the 



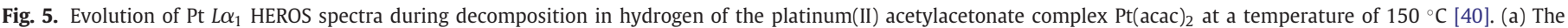

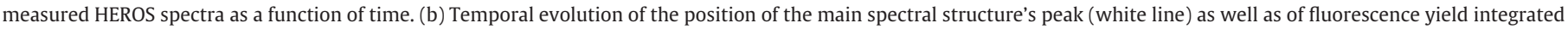

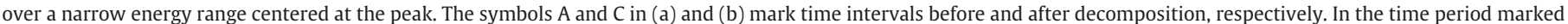
with B an intermediate step in the starting complex' transition was observed. The figure was reproduced from Ref. [40].

dissociative adsorption of oxygen at the Pt surface, and the second step is the partial oxidation of the Pt-subsurface by oxide diffusion. It was shown, that the intermediate adsorption could be described as a $\mathrm{O}$-atop position chemisorption process on a metallic surface.

\subsubsection{In situ study on silica supported Ta catalyst activation and oxidation}

The high chemical sensitivity and swiftness of the HEROS approach found also application in a study on temporal changes of the density of unoccupied states of Ta atoms in two different silica supported Ta catalysts during their oxidation [49,50]. The measurements were performed at a synchrotron for two Ta catalysts: inactive Ta(V) bisalkyl complex $\left[\left(\equiv \mathrm{SiO}_{2}\right) \mathrm{Ta}(=\mathrm{CH} t \mathrm{Bu})\left(\mathrm{CH}_{2} t \mathrm{Bu}\right)_{2}\right][49]$ and the same complex activated in hydrogen [50]. The studied target material was placed in a quartz capillary reactor cell in a glove box under to $20 \mathrm{ppm} \mathrm{O}_{2}$ atmosphere and at room. The target was irradiated with a monochromatic synchrotron beam of energy fixed below Ta $L_{3}$-edge binding energy. By means of a von Hamos geometry-based spectrometer, the Ta $L \alpha_{1}$ HEROS spectra were recorded one by one with acquisition time of $40 \mathrm{~s}$. To obtain the activated $\mathrm{Ta}(\mathrm{V})$ bisalkyl complex, the inactive complex was exposed to $10 \% \mathrm{H}_{2} / \mathrm{He}$ at the temperature of $150{ }^{\circ} \mathrm{C}$.

For both complexes studied the oxidation resulted in a change of the intensity, energy position and shape of the spectral structures observed in the HEROS spectra. Spectral analysis, supported with calculations done with FEFF software [72] and the Kramers-Heisenberg formula modified by Tulkki and Åberg [30,31], revealed that oxidation of the inactive and the active Ta catalyst leads to the formation of mono- and di-meric Ta species on the $\mathrm{SiO}_{2}$ surface. It was identified that the electronic states with the largest contributing to the HEROS signal originated from the Ta $d$-band. Moreover, using the fingerprint HEROS spectra measured for each complex before and after its reaction with oxygen, the temporal evolution of the relative species' concentration was successfully retrieved as presented in Fig. 7. It allowed observation that the inactive Ta catalyst's transition from its unoxidized to the oxidized form has a stepwise character, probably due to different rates for oxidation and dimerization.

\subsubsection{An operando study on $\mathrm{Pt} / \mathrm{Al}_{2} \mathrm{O}_{3}$ and $\mathrm{Pt} / \mathrm{CeO}_{2} / \mathrm{Al}_{2} \mathrm{O}_{3}$}

In a further development of the HEROS methodology, the experimental time-resolved data were evaluated with analysis tools based on phase sensitive detection (PSD) [45]. The methodology was applied to in situ time-resolved studies of catalysis based on dispersed nanoparticles on metal oxides. Such a system facilitates to clarify and to follow reaction mechanisms at transient conditions. HEROS was first applied to follow the temperature-programmed reduction of 1.3 wt. \% $\mathrm{Pt} / \mathrm{Al}_{2} \mathrm{O}_{3}$ and 1.3 wt. \% Pt/20 wt. \% $\mathrm{CeO}_{2} / \mathrm{Al}_{2} \mathrm{O}_{2}$ catalysts. Comparison of time-resolved HEROS and XANES data allowed to demonstrate the increased sensitivity and time resolution of the HEROS technique in this type of experiments. It was shown, that enhanced sensitivity of off-resonant measurements can be further improved by modulation excitation spectroscopy. This approach explores the fact that time-resolved HEROS data acquisition is performed over repeated gas pulses applied to catalysis in order to increase the signal-to-noise ratio of the spectroscopic signal. HEROS spectra thus can be treated with frequency response mathematical tools such as phase sensitive detection (PSD). In this way, the averaged time-resolved spectra can be transformed into a set of phase-resolved spectra, and therefore the contributions of the species that are not responding to the external stimulation (like gas switches or temperature ramps) are removed. The phaseresolved spectra exhibit thus an enhanced signal-to-noise ratio. Moreover, HEROS-PSD facilitates identification of intermediate components because PSD-transformed spectra contain the information only about the species that react to applied external changes. Similar approaches were already applied to infrared spectroscopy [73-75], X-ray absorption spectroscopy [76-78] and X-ray diffraction [79,80] experiments proving that an additional level of information can be obtained. In this paper, the PSD methodology in combination with HEROS was confirmed and its improved sensitivity to the structural and electronic changes of probed material was demonstrated.

The PSD-HEROS methodology was thus employed to follow reversible oxidation-reduction processes on $\mathrm{Pt}$ nanoparticles at $300{ }^{\circ} \mathrm{C}$ in modulation experiments consisting of alternate pulses of $\mathrm{H}_{2}$ and $\mathrm{O}_{2}$ at $300{ }^{\circ} \mathrm{C}$. Examples of time-resolved and phase-resolved HEROS spectra are plotted in Fig. 8. It was clearly shown that the PSD approach is necessary to obtain enhanced sensitivity to the Pt-O intermediate species at high gas feed concentrations. Fraction of oxidized $\mathrm{Pt}$ in the $\mathrm{H}_{2}-\mathrm{O}_{2}$ modulation was clearly increased by adding $\mathrm{CeO}_{2}$ particles. Similar observations were drawn in $\mathrm{CO}-\mathrm{O}_{2}$ gas modulation experiments that allow identifying molecule adsorption geometry on metal nanoparticles as Pt-CO and the atop Pt-O. The experiments demonstrated that HEROS is a powerful in situ/operando spectroscopy method with increased sensitivity by applying modulation analysis. The unoccupied electronic states of matter can be accessed with excellent time- and energy-resolution, as compared to conventional XANES experiments.

\subsection{HEROS with pulsed sources}

The HEROS technique was first introduced at synchrotron radiation sources, where high intensity and highly monochromatic X-ray 




Fig. 6. The data measured with HEROS during reduction and reoxidation of $\mathrm{Pt} / \mathrm{Al}_{2} \mathrm{O}_{3}$ catalyst [43]. (a) Evolution of the intensity integrated over two narrow energy ranges centered at $9.426 \mathrm{keV}$ and $9.427 \mathrm{keV}$. (b) Pt $L \alpha_{1}$ HEROS spectra measured in three moments during reoxidation, marked with dashed arrows in (a). The dashed lines in (b) indicate the two integration energy ranges. The figure was reproduced from Ref. [43].

radiation was available $[40,43,49,81]$. Indeed, the monochromatic radiation allows for a precise detuning with respect to the elemental core level binding energy under investigation, thus to fulfill the experimental requirement for investigations by means of HEROS. Using furthermore a dispersive-type high-energy resolution emission spectrometer with a sufficient energy bandwidth, e.g., a von Hamos spectrometer, HEROS measurements can be performed in a scanningfree manner with a time-resolution being only limited by the incident photon flux and the detection efficiency. This makes the HEROS technique highly suitable for pulsed X-ray sources, like X-ray free electron lasers (XFELs) since through the Kramers-Heisenberg formalism [31] X-ray absorption-like information $[41,44]$ can be obtained in a singleshot fashion. Note that in contrast to XAS the incident photon energy is kept fixed in a HEROS experiment and the shape of the emitted spectrum is recorded in a single acquisition. Single-shot XAS, on the other hand, is usually difficult to realize because of the limited energy bandwidth offered by pulsed X-ray sources. Scanning the incident photon energy requires, furthermore, that the incident beam properties in terms of pulse energy, pulse duration and photon flux are monitored with high precision for later normalization purposes.

A first HEROS experiment at a XFEL operated in the self-seeded mode [82] was performed on $\mathrm{Cu}$ and different $\mathrm{Cu}$ oxides around the $\mathrm{Cu}$ K-edge [48]. A von Hamos spectrometer operated in a multi-crystal arrangement was used to monitor simultaneously the signals from the $K \alpha$ and the $K \beta$ emission lines [83] (see Fig. 9 (a)). The shape of the HEROS spectra, acquired entirely on a shot-to-shot basis, was found to be independent of the intensity fluctuations of the source for fixed
X-ray beam parameters meaning that the information acquired through HEROS is insensitive to pulse-to-pulse fluctuations. Furthermore, the chemical sensitivity of the HEROS technique was proven through the energy shifts of the HEROS signal which were directly connected to the oxidation state of the probed atoms. The main characteristic features of the HEROS spectra were already identifiable after a single XFEL pulse. Thus, it was demonstrated that for solid samples HEROS presents, despite the low scattering and absorption cross sections, the potential for element-selective single-shot studies of the electronic and structural properties of matter using femtosecondduration pulses by probing the density of unoccupied states.

The pulse duration aspect is noteworthy since, thanks to the selfseeding scheme of the XFEL, any temporal pulse broadening due to the finite extinction length of the X-rays into the monochromator crystal could be avoided [84]. Considering the high peak power of the radiation provided by XFELs which allows for non-linear X-ray spectroscopy studies, HEROS was also found to be a useful tool for the study of the two-photon absorption (TPA) mechanism [47]: when increasing the X-ray fluence on the sample, the non-resonant $\mathrm{Cu} \mathrm{K \alpha} \alpha_{1}$ and $K \alpha_{2} \mathrm{X}$-ray emission emerged from the HEROS spectrum as a result of a two photon X-ray absorption process. Besides the determination of the cross section and the fluence threshold of the TPA process, HEROS might prove in future to be useful for the study of quadrupole transitions through an X-ray optical wave mixing scheme [47]. Finally, HEROS experiments are complementary to non-resonant XES experiments which can also be conducted in a single-shot scheme at pulsed $\mathrm{X}$-ray sources to probe the density of occupied states. The incident
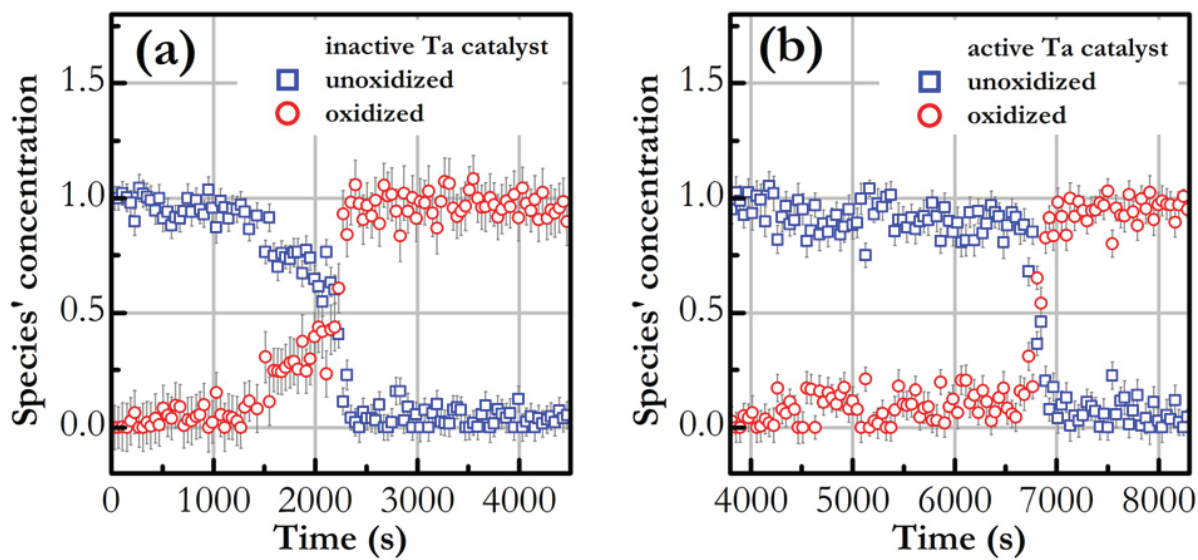

Fig. 7. Relative concentration of inactive Ta(V) bisalkyl complex [49] (a) and the one activated in hydrogen [50] (b) as a function of time during oxidation. The intermediate step in oxidation of the inactive Ta catalyst was not observed during the active Ta catalyst oxidation. The figure was reproduced from Ref. [50]. 

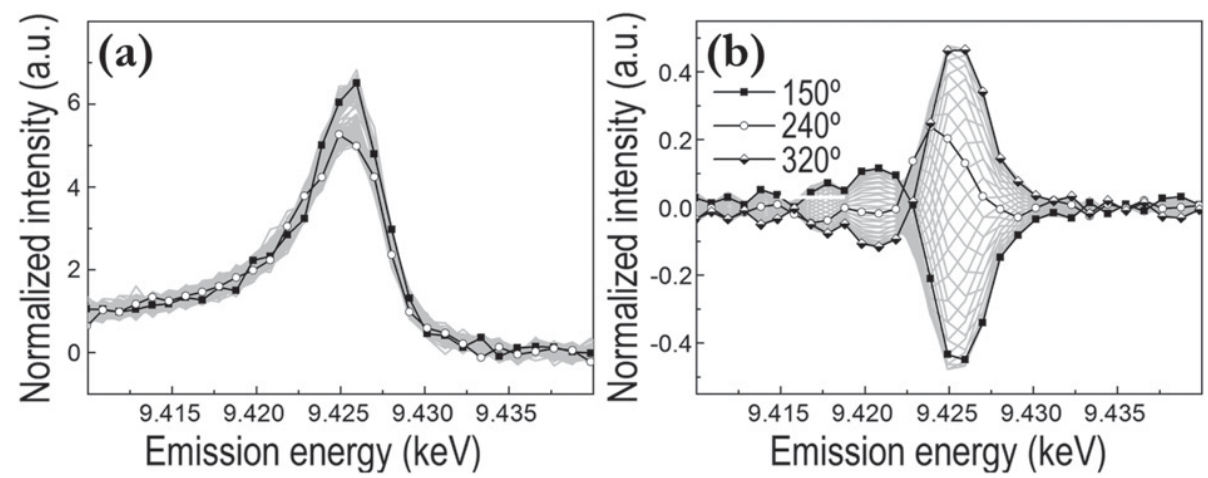

Fig. 8. Time-resolved (a) and phase-resolved (b) HEROS spectra collected at the Pt $L_{3}$ edge during $1 \mathrm{vol} . \% \mathrm{CO} / \mathrm{He}-1$ vol.\% $\mathrm{O}_{2} / \mathrm{He}$ modulation experiment on Pt/Al $\mathrm{O}_{2}$ at $300{ }^{\circ} \mathrm{C}[45]$. Full markers in: last spectrum in $\mathrm{O}_{2}$, open markers: last spectrum in CO. The figure was reproduced from Ref. [45].

photon energy only needs to be tuned above the ionization threshold probed by means of HEROS. Both HEROS and XES are quite insensitive to self-absorption effects, thus to sample concentration and thickness effects. Together, HEROS and XES provide then a complete picture of the electronic state of the system under investigation. Prospectively, the single-shot aspect of HEROS makes the technique very attractive for pump-probe experiments at pulsed X-ray sources in order to follow, possibly under in situ conditions, time-dependent changes of the electronic structure. In this view it should also be noted that HEROS measurements can be conducted in parallel with diffraction or scattering experiments.

\section{Conclusions and outlook}

HEROS is an element specific and scanning free methodology deprived of self-absorption events that profits from the high penetration depth of X-rays to map rigorously the absorbable atom's density of states. The aforementioned properties makes HEROS studies particularly suited for time-resolved spectroscopic studies under relevant working conditions, which is veracious and relevant for a plethora of research fields, including all kinds of catalysis, material science, inorganic chemistry including metallo-based therapies, biology, physics, etc. With respect to future perspectives, we consider that HEROS will be a champion methodology to study reactivity in two temporal regimes, namely the ultrafast and the kinetic-controlled domain. Some of these applications have been already demonstrated or are being commissioned, which substantiates the present claim. To truly benefit from HEROS properties in both temporal regimes one must devise selective and controllable triggers specific for each experiment and field of study. In the ultrafast domain, the classical optical triggers will be the most common and possibly the easiest approach. They allow the study of photo-activated processes relevant for solar

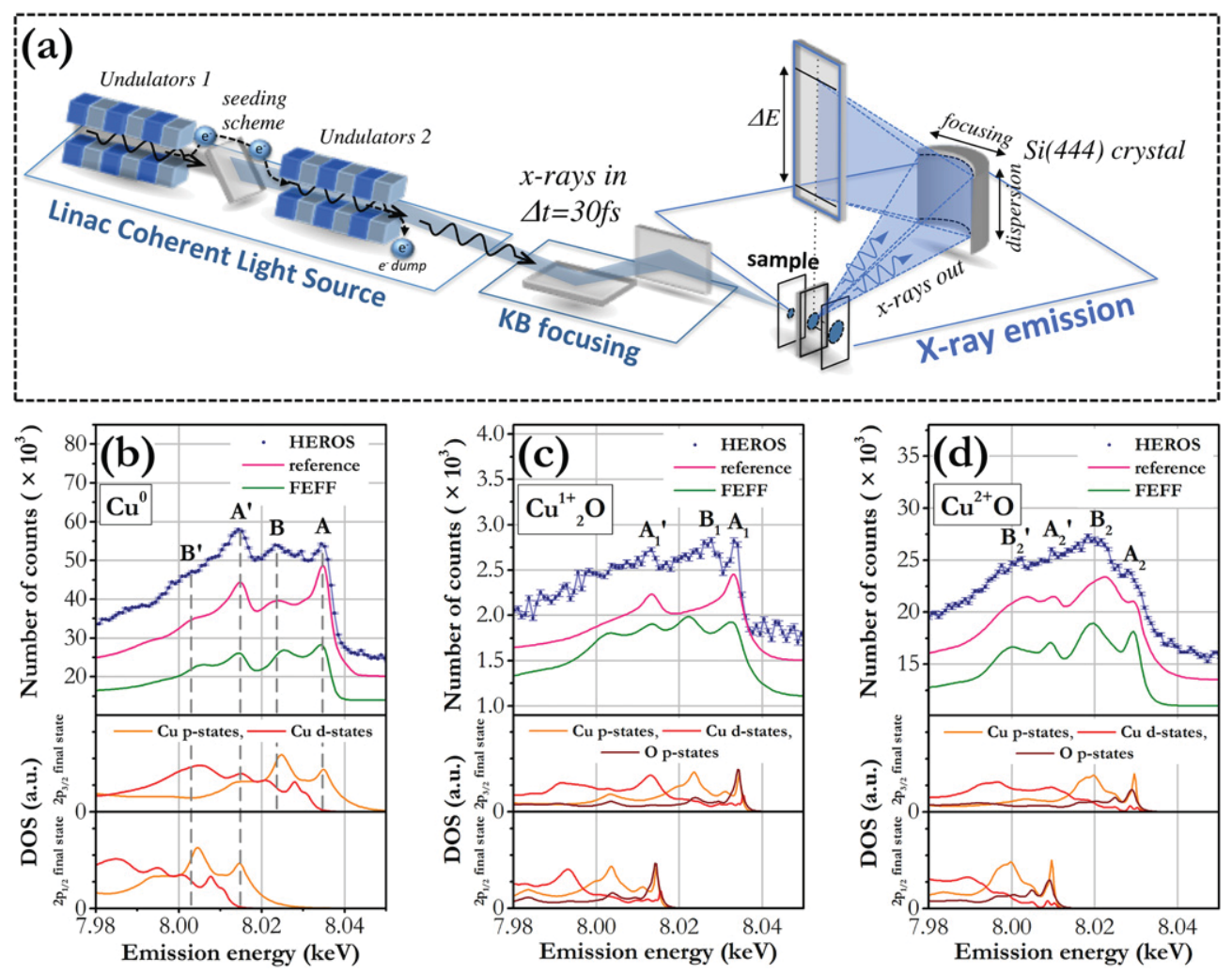

Fig. 9. (a) Illustration of the experimental setup used in the study. The HEROS spectra presented in (b), (d) [48] and (c) were measured for $\mathrm{Cu}_{\text {foil, }} \mathrm{CuO}_{\text {and }} \mathrm{Cu}_{2} \mathrm{O}$, respectively. For comparison, calculations based on the reference XAS spectra (in pink) and the theoretical XAS spectra (by FEFF; in green) are presented. The lower panels show the density of the most contributing unoccupied states (by FEFF) as a function of the energy of the emitted photons. Note additional $\mathrm{O} p$-states contributing to the off-resonant emission spectra in the case of the two copper oxides. The data in (b) and (d) come from Ref. [48]. 
applications, photo-therapy, among others. However, it excludes the study of systems that do not have selective absorptions, which includes näst of the catalytic processes. To enable the study of such processes, one must use more elaborated pumping schemes with alternative radiation, such as terahertz, and/or multi-color and shaped optical pulses. In the kinetic-controlled domain, the selective triggers have to be connected to changing reaction conditions, i.e., varying parameters such as temperature, chemical composition, exposed magnetic field. Some are easier to be achieved than others but the approach explores the fact that time-resolved HEROS data acquisition is accomplished over repeated switches, which increases the signal-to-noise ratio of the spectroscopic signal. Moreover, the HEROS spectra can be treated with frequency response mathematical tools, such as phase or frequency sensitive detection, which provides all important kinetic information. Currently, we cannot see potential limitations to the use of HEROS methodology in time-resolved spectroscopic studies under relevant working conditions. However, HEROS studies are on its embryonic phase and only its expansion can highlight potential limitations that are currently not observable.

\section{Acknowledgments}

The authors thank the Swiss Light Source at the Paul Scherrer Institute, Switzerland for access to the SuperXAS beam line and available laboratories. Use of the Linac Coherent Light Source (LCLS), SLAC National Accelerator Laboratory, is supported by the U.S. Department of Energy, Office of Science, Office of Basic Energy Sciences under contract no. DE-AC02-76SF00515. W. B., J. H. and J.-Cl. D. acknowledge the Swiss National Science Foundation for the financial support, grant no. 200020-146739. W. B., J. Sá and J. Sz. acknowledge the Polish Ministry of Science and Higher Education for support from the budget allocations for science for years 2016-2019 within the IDEAS PLUS II project of number IdPII2015000164. J. Sz. acknowledges National Science Centre, Poland (NCN) for support under grant nos. 2015/18/E/ST3/00444 and 2015/19/B/ST2/00931. We also wish to thank Dr. Szeto, Dr. Mallmann and Dr. Taoufik from CNRS-LCOMS, Université Lyon 1 who prepared the Ta catalyst samples and helped us with their specialized knowledge.

\section{References}

[1] C. Bonnelle, X-Ray Spectroscopy, Annu. Rep. Prog. Chem., Sect. C: Phys. Chem 84 (1987) 201-272. http://dx.doi.org/10.1039/PC9878400201.

[2] E.P. Bertin, Principles and Practice of X ray Spectrometric Analysis, second edition ed., Plenum Press, New York, 1975.

[3] Y. Cauchois, Les niveaux d'énergie des atomes de numéro atomique inférieur á 70, J. Phys. Radium 16 (1955) 253-262. http://dx.doi.org/10.1051/jphysrad: 01955001604025300.

[4] B. Crasemann (Ed.), Atomic Inner-Shell Processes, Academic Press.1975.

[5] TFR Group, M. Cornille, J. Dubau, M. Loulergue, Charge-dependent wavelength shifts and line intensities in the dielectronic satellite spectrum of heliumlike ions, Phys. Rev. A 32 (1985) 3000. http://dx.doi.org/10.1103/PhysRevA.32.3000.

[6] A. Warland, C. Antoniak, M. Darbandi, C. Weis, J. Landers, W. Keune, $\mathrm{H}$. Wende, Effect of silica capping on the oxidation of $\mathrm{Fe}_{3} \mathrm{O}_{4}$ nanoparticles in dispersion revealed by x-ray absorption spectroscopy, Phys. Rev. B 85 (2012) 235113. http://dx.doi.org/10.1103/PhysRevB.85.235113.

[7] S. Landsberger, C. Brabec, B. Canion, J. Hashem, C. Lu, D. Millsap, G. George, Determination of ${ }^{226} \mathrm{Ra},{ }^{228} \mathrm{Ra}$ and ${ }^{210} \mathrm{~Pb}$ in NORM products from oil and gas exploration: problems in activity underestimation due to the presence of metals and self-absorption of photons, J. Environ. Radioact. 125 (2013) 23-26. http://dx.doi.org/10.1016/j.jenvrad.2013.02.012.

[8] M. Gräfe, E. Donner, R.N. Collins, E. Lombi, Speciation of metal(loid)s in environmental samples by X-ray absorption spectroscopy: a critical review, Anal. Chim. Acta 822 (2014) 1-22. http://dx.doi.org/10.1016/j.aca.2014.02.044.

[9] R. Ortega, A. Carmona, I. Llorens, P.L. Solari, X-ray absorption spectroscopy of biological samples. A tutorial, J. Anal. At. Spectrom. 27 (2012) 2054-2065. http://dx.doi.org/10.1039/C2JA30224A.

[10] S. Bordiga, E. Groppo, G. Agostini, J.A. van Bokhoven, C. Lamberti, Reactivity of surface species in heterogeneous catalysts probed by in situ X-ray absorption techniques, Chem. Rev. 113 (2013) 1736-1850. http://dx.doi.org/10.1021/ cr2000898.

[11] X. Chen, et al. Properties of disorder-engineered black titanium dioxide nanoparticles through hydrogenation, Sci. Rep. 3 (2013) 1510. http://dx.doi. org/10.1038/srep01510.
[12] R. Mitzner, et al. L-edge X-ray absorption spectroscopy of dilute systems relevant to metalloproteins using an X-ray free-electron laser, J. Phys. Chem. Lett. 4 (2013) 3641-3647. http://dx.doi.org/10.1021/jz401837f.

[13] D.S. Urch, X-ray emission spectroscopy, Q. Rev. Chem. Soc. 25 (1971) 343-364 http://dx.doi.org/10.1039/QR9712500343.

[14] U. Bergmann, P. Glatzel, X-ray emission spectroscopy, Photosynth. Res. 102 (2009) 255-266. http://dx.doi.org/10.1007/s11120-009-9483-6.

[15] U. Bergmann, C.R. Horne, T.J. Collins, J.M. Workman, S.P. Cramer, Chemical dependence of interatomic X-ray transition energies and intensities - a study of Mn $K \beta_{\prime \prime}$ and $K \beta_{2,5}$ spectra, Chem. Phys. Lett. 302 (1999) 119-124. http://dx. doi.org/10.1016/S0009-2614(99)00095-0.

[16] V.A. Safonov, et al. Valence-to-core X-ray emission spectroscopy identification of carbide compounds in nanocrystalline $\mathrm{Cr}$ coatings deposited from $\mathrm{Cr}(\mathrm{III})$ electrolytes containing organic substances, J. Phys. Chem. B 110 (2006) 23192-23196. http://dx.doi.org/10.1021/jp064569j.

[17] C.J. Pollock, S. DeBeer, Valence-to-core X-ray emission spectroscopy: a sensitive probe of the nature of a bound ligand, J. Am. Chem. Soc. 133 (2011) 5594-5601. http://dx.doi.org/10.1021/ja200560z.

[18] F.W. Lytle, X-ray absorption spectroscopy, Berichte der Bunsengesellschaft für physikalische Chemie, 91. 1987, 1251-1257. http://dx.doi.org/10.1002/bbpc. 19870911134

[19] J. Yano, V.K. Yachandra, X-ray absorption spectroscopy, Photosynth. Res. 102 (2009) 241-254. http://dx.doi.org/10.1007/s11120-009-9473-8.

[20] J. Jaklevic, J.A. Kirby, M.P. Klein, A.S. Robertson, G.S. Brown, P. Eisenberger, Fluorescence detection of exafs: sensitivity enhancement for dilute species and thin films, Solid State Commun. 23 (1977) 679-682. http://dx.doi.org/10.1016/ 0038-1098(77)90548-8.

[21] W. Gudat, C. Kunz, Close similarity between photoelectric yield and photoabsorption spectra in the soft-X-ray range, Phys. Rev. Lett 29 (1972) 169. http:// dx.doi.org/10.1103/PhysRevLett.29.169.

[22] A. Erbil, G.S. Cargill, III, R. Frahm, R.F. Boehme, Total-electron-yield current measurements for near-surface extended x-ray-absorption fine structure, Phys. Rev. B 37 (1988) 2450. http://dx.doi.org/10.1103/PhysRevB.37.2450.

[23] P. Eisenberger, P.M. Platzman, H. Winick, X-ray resonant Raman scattering: observation of characteristic radiation narrower than the lifetime width, Phys Rev. Lett. 36 (1976) 623. http://dx.doi.org/10.1103/PhysRevLett.36.623.

[24] K. Hämäläinen, D.P. Siddons, J.B. Hastings, L.E. Berman, Elimination of the inner-shell lifetime broadening in x-ray-absorption spectroscopy, Phys. Rev. Lett. 67 (1991) 2850. http://dx.doi.org/10.1103/PhysRevLett.67.2850.

[25] F. Reinhardt, et al. Evaluation of high-resolution X-ray absorption and emission spectroscopy for the chemical speciation of binary titanium compounds, Anal. Chem. 81 (2009) 1770-1776. http://dx.doi.org/10.1021/ac8018069.

[26] A. Kotani, S. Shin, Resonant inelastic x-ray scattering spectra for electrons in solids, Rev. Mod. Phys. 73 (2001) 203. http://dx.doi.org/10.1103/RevModPhys 73.203.

[27] P. Glatzel, et al. Hard x-ray photon-in-photon-out spectroscopy with lifetime resolution - of XAS, XES, RIXSS and HERFD, AIP Conf. Proc. 879 (2007) 1731 http://dx.doi.org/10.1063/1.2436402.

[28] J. Singh, C. Lamberti, J.A. van Bokhoven, Advanced X-ray absorption and emission spectroscopy: in situ catalytic studies, Chem. Soc. Rev 39 (2010) 4754-4766. http://dx.doi.org/10.1039/C0CS00054J.

[29] C.J. Sparks, Jr, Inelastic resonance emission of X rays: anomalous scattering associated with anomalous dispersion, Phys. Rev. Lett. 33 (1974) 262. http://dx. doi.org/10.1103/PhysRevLett.33.262.

[30] J. Tulkki, T. Åberg, Behaviour of Raman resonance scattering across the K X-ray absorption edge, J. Phys. B: At. Mol. Phys. 15 (1982) L435-L440. http://dx.doi. org/10.1088/0022-3700/15/13/004.

[31] H.A. Kramers, W. Heisenberg, Über die Streuung von Strahlung durch Atome, Z. Phys. 31 (1925) 681-708. http://dx.doi.org/10.1007/BF02980624

[32] P. Suortti, V. Eteläniemi, K. Hämäläinen, S. Manninen, Fine structure of resonant Raman scattering, J. Phys. Colloques 48 (1987) c9-831-C9-834. http://dx. doi.org/10.1051/jphyscol:19879147.

[33] V. Eteläniemi, K. Hämäläinen, S. Manninen, P. Suortti, XANES spectroscopy using high-resolution resonant Raman scattering: application to holmium, J. Phys.: Condens. Matter 4 (1992) 879-886. http://dx.doi.org/10.1088/09538984/4/3/027.

[34] H. Hayashi, R. Takeda, Y. Udagawa, T. Nakamura, H. Miyagawa, H. Shoji, S. Nanao, N. Kawamura, Lifetime-broadening-suppressed/free XANES spectroscopy by high-resolution resonant inelastic X-ray scattering, Phys. Rev. B 68 (2003) 045122. http://dx.doi.org/10.1103/PhysRevB.68.045122.

[35] H. Hayashi, R. Takeda, Y. Udagawa, T. Nakamura, H. Miyagawa, H. Shoji, S. Nanao, N. Kawamura, Lifetime-broadening-removed XANES spectroscopy by high-resolution resonant inelastic x-ray scattering, Phys. Scr. T115 (2005) 1094-1096. http://dx.doi.org/10.1238/Physica.Topical.115a01094.

[36] J. Szlachetko, J.-Cl. Dousse, J. Hoszowska, M. Pajek, R. Barrett, M. Berset, K. Fennane, A. Kubala-Kukus, M. Szlachetko, High-resolution study of X-ray resonant Raman scattering at the K edge of silicon, Phys. Rev. Lett 97 (2006) 073001. http://dx.doi.org/10.1103/PhysRevLett.97.073001.

[37] J. Szlachetko, J.-Cl. Dousse, M. Berset, K. Fennane, M. Szlachetko, J. Hoszowska, R. Barrett, M. Pajek, A. Kubala-Kukus, High-resolution study of the $\mathrm{X}$-ray resonant Raman scattering process around the $1 \mathrm{~s}$ absorption edge for aluminium, silicon, and their oxides, Phys. Rev. A 75 (2007) 022512. http://dx.doi. org/10.1103/PhysRevA.75.022512.

[38] D.T. Bowron, M.H. Krisch, A.C. Barnes, J.L. Finney, A. Kaprolat, M. Lorenzen, $\mathrm{X}$-ray-Raman scattering from the oxygen $\mathrm{K}$ edge in liquid and $\mathrm{H}_{2} \mathrm{O}$, Phys. Rev. B 62 (2000) R9223. http://dx.doi.org/10.1103/PhysRevB.62.R9223. 
[39] D. Sokaras, et al. A high resolution and large solid angle x-ray Raman spectroscopy end-station at the Stanford Synchrotron Radiation Lightsource, Rev. Sci. Instrum. 83 (2012) 043112. http://dx.doi.org/10.1063/1.4704458.

33 [40] J. Szlachetko, et al. High energy resolution off-resonant spectroscopy at subsecond time resolution: (Pt(acac) $)_{2}$ ) decomposition, Chem. Commun. 48 (2012) 10898-10900. http://dx.doi.org/10.1039/C2CC35086F.

[41] M. Kavčič, M. žitnik, K. Bučar, A. Mihelič, B. Marolt, J. Szlachetko, P. Glatzel, K. Kvashnina, Hard x-ray absorption spectroscopy for pulsed sources, Phys. Rev. B 87 (2013) 075106. http://dx.doi.org/10.1103/PhysRevB.87.075106.

[42] J. Sá (Ed.), High-resolution XAS/XES: analyzing electronic structures of catalysts, CRC Press.2014,Ch. Techniques: RXES, HR-XAS, HEROS, GIXRF, and GEXRF, ISBN 978-1-4665-9298-8.

[43] J. Szlachetko, D. Ferri, V. Marchionni, A. Kambolis, O.V. Safonova, C.J. Milne, O. Kröcher, M. Nachtegaal, J. Sá, Subsecond and in situ chemical speciation of $\mathrm{Pt} / \mathrm{Al}_{2} \mathrm{O}_{3}$ during oxidation reduction cycles monitored by high-energy resolution off-resonant X-ray spectroscopy, J. Am. Chem. Soc. 135 (2013) 19071-19074. http://dx.doi.org/10.1021/ja410146c.

[44] W. Błachucki, J. Szlachetko, J. Hoszowska, J.-Cl. Dousse, Y. Kayser, M. Nachtegaal, J. Sá, High energy resolution off-resonant spectroscopy for X-ray absorption spectra free of self-absorption effects, Phys. Rev. Lett. 112 (2014) 173003. http://dx.doi.org/10.1103/PhysRevLett.112.173003.

[45] V. Marchionni, J. Szlachetko, M. Nachtegaal, A. Kambolis, O. Kröcher, D. Ferri, An operando emission spectroscopy study of $\mathrm{Pt} / \mathrm{Al}_{2} \mathrm{O}_{3}$ and $\mathrm{Pt} / \mathrm{CeO}_{2} / \mathrm{Al}_{2} \mathrm{O}_{3}$, Phys. Chem. Chem. Phys. 18 (2016) 29268-29277. http://dx.doi.org/10.1039/ C6CP05992A

[46] R. Kopelent, J.A. van Bokhoven, M. Nachtegaal, J. Szlachetko, O.V. Safonova, $\mathrm{X}$-ray emission spectroscopy: highly sensitive techniques for time-resolved probing of cerium reactivity under catalytic conditions, Phys. Chem. Chem. Phys. 18 (2016) 32486-32493. http://dx.doi.org/10.1039/C6СР05830B.

[47] J. Szlachetko, J. Hoszowska, J.-Cl. Dousse, M. Nachtegaal, W. Błachucki, Y. Kayser, J. Sá, M. Messerschmidt, S. Boutet, G.J. Williams, C. David, G. Smolentsev, J.A. van Bokhoven, B.D. Patterson, T.J. Penfold, G. Knopp, M. Pajek, R Abela, C.J. Milne, Establishing nonlinearity thresholds with ultraintense X-ray pulses, Sci. Rep. 6 (2016) 33292. http://dx.doi.org/10.1038/srep33292.

[48] J. Szlachetko, et al. The electronic structure of matter probed with a single femtosecond hard x-ray pulse, Struct. Dyn. 1 (2014) 021101. http://dx.doi.org/10. $1063 / 1.4868260$.

[49] W. Błachucki, J. Szlachetko, Y. Kayser, J.-Cl. Dousse, J. Hoszowska, D.L.A Fernandes, J. Sá, Study of the reactivity of silica supported tantalum catalysts with oxygen followed by in situ HEROS, Phys. Chem. Chem. Phys. 17 (2015) 18262-18264. http://dx.doi.org/10.1039/c5cp02950c.

[50] W. Błachucki, J. Szlachetko, Y. Kayser, J.-C. Dousse, J. Hoszowska, F. Zeeshan, J. Sá, Insitu high energy resolution off-resonant spectroscopy applied to a time-resolved study of single site Ta catalyst during oxidation, Nucl. Instr. Meth. Phys. Res. B. (2016) http://dx.doi.org/10.1016/j.nimb.2016.11.029.

[51] J. Sá, In situ time-resolved HEROS study of Pt electronic structure during regenerative $\mathrm{H}_{2}-\mathrm{O}_{2}$ cycles, Recycl. Catal 2 (2015) 23-26. http://dx.doi.org/10.1515 recat-2015-0004

[52] J.A. Van Bokhoven, C. Lamberti (Eds.), X-Ray Absorption and X-Ray Emission Spectroscopy: Theory and Applications, John Wiley \& Sons, Ltd, Chichester, UK, 2016, Ch. X-Ray Spectroscopy at Free Electron Lasers. http://dx.doi.org/10 $1002 / 9781118844243 . c h 22$

[53] L.E. Norena, J.A. Wang (Eds.), Advanced Catalytic Materials - Photocatalysis and Other Current Trends, InTech, 2016, Ch. X-Ray Spectroscopy - The Driving Force to Understand and Develop Catalysis. http://dx.doi.org/10.5772/61940.

[54] B.D. Patterson, P. Beaud, H.H. Braun, C. Dejoie, G. Ingold, C. Milne, L. Patthey, B. Pedrini, J. Szlachetko, R. Abela, Science opportunities at the SwissFEL X-ray Laser, CHIMIA Int. J. Chem. 68 (2014) 73-78. http://dx.doi.org/10.2533/chimia.

[55] C.J. Milne, T.J. Penfold, M. Chergui, Recent experimental and theoretical developments in time-resolved X-ray spectroscopies, Coordination Chemistry Reviews 277-278 (2014) 44-68. http://dx.doi.org/10.1016/j.ccr.2014.02.013.

[56] O. Müller, M. Nachtegaal, J. Just, D. Lützenkirchen-Hechta, R. Frahma, Quick-EXAFS setup at the SuperXAS beamline for in situ X-ray absorption spectroscopy with 10 ms time resolution, J. Synchrotron Radiat. 23 (2016) 260-266. http://dx.doi.org/10.1107/S1600577515018007.

[57] M. Chergui, Time-resolved X-ray spectroscopies of chemical systems: new perspectives, Struct. Dynam. 3 (2016) 031001. http://dx.doi.org/10.1063/1. 4953104.

[58] M. žitnik, M. Kavčič, K. Buăr, A. Mihelič, R. Bohinc, New results in highresolution X-ray fluorescence spectroscopy, J. Phys. Conf. Ser. 488 (2014) 012014. http://dx.doi.org/10.1088/1742-6596/488/1/012014.

[59] M. žitnik, M. Kavčič, R. Bohinc, K. Bučar, A. Mihelič, W. Cao, R. Guillemin, L. Journel, T. Marchenko, S. Carniato, E. Kawerk, M.N. Piancastelli, M. Simon, Resonant inelastic X-ray spectroscopy of atoms and simple molecules: satellite features and dependence on energy detuning and photon polarization, J. Electron Spectrosc. Relat. Phenom. 204 (2015) 356-364. http://dx.doi.org/10.1016/ j.elspec.2015.05.018.

[60] M.A. Newton, Time resolved operando X-ray techniques in catalysis, a case study: $\mathrm{CO}$ oxidation by $\mathrm{O}_{2}$ over Pt surfaces and alumina supported Pt catalysts, Catalysts 7 (2017) 58. http://dx.doi.org/10.3390/catal7020058.

[61] A. Bagger, T. Haarman, A. Puig Molina, P.G. Moses, H. Ishii, N. Hiraoka, Y.H. Wu, K.D. Tsuei, I. Chorkendorff, F. De Groot, $11 \mathrm{~s} 2$ p resonant inelastic X-ray scattering combined dipole and quadrupole analysis method, J. Synchrotron Rad. 24 (2017) 296-301. http://dx.doi.org/10.1107/S1600577516016933.
[62] N.E. Tsakoumis, A.P.E. York, D. Chen, M. Rønning, Catalyst characterisation techniques, reaction cells operating at realistic conditions; towards acquisition of kinetically relevant information, Catal. Sci Technol. 5 (2015) 4859-4883. http://dx.doi.org/10.1039/C5CY00269A.

[63] S.A. Bartlett, M.L. Hamilton, J. Evans, Dynamic structure elucidation of chemical reactivity by laser pulses and X-ray probes, Dalton Trans. 44 (2015) 6313-6319. http://dx.doi.org/10.1039/C5DT00210A.

[64] S.A. Bartlett, M.L. Hamilton, J. Evans, S.A. Bartlett, M.L. Hamilton, J. Evans, Ce K edge XAS of ceria-based redox materials under realistic conditions for the two-step solar thermochemical dissociation of water and/or $\mathrm{CO}_{2}$, Phys. Chem. Chem. Phys 17 (2015) 26988-26996. http://dx.doi.org/10.1039/C5CP03179F.

[65] C. Vettier, Resonant elastic X-ray scattering: where from? Where to? Eur. Phys. J. Special Topics 208 (2012) 3-14. http://dx.doi.org/10.1140/epjst/e201201602-7.

[66] J. Fink, E. Schierle, E. Weschke, J. Geck, Resonant elastic soft x-ray scattering, Rep. Prog. Phys. 76 (2013) 056502. http://dx.doi.org/10.1088/0034-4885/76/5/ 056502.

[67] L.J.P. Ament, M. van Veenendaal, T.P. Devereaux, J.P. Hill, J. van den Brink, Resonant inelastic x-ray scattering studies of elementary excitations, Rev. Mod. Phys. 83 (2011) 705. http://dx.doi.org/10.1103/RevModPhys.83.705.

[68] M. Simon, T. Schmitt (Eds.), Special issue on Progress In Resonant Inelastic X-ray scattering, 2013,J. Electron Spectrosc. Rel. Phenom., ISSN 0368-2048.

[69] P.A.M. Dirac, The quantum theory of dispersion, Proc. R. Soc. Lond. A 114 (1927) 710-728. http://dx.doi.org/10.1098/rspa.1927.0071.

[70] S. Eisebitt, T. Böske, J.E. Rubensson, W. Eberhardt, Determination of absorption coefficients for concentrated samples by fluorescence detection, Phys. Rev. B 47 (1993) 14103. http://dx.doi.org/10.1103/PhysRevB.47.14103.

[71] S.G. Fiddy, et al. Particle development and characterisation in Pt(acac) 2 and $\mathrm{Pt}(\mathrm{acac})_{2} / \mathrm{GeBu}_{4}$ derived catalysts supported upon porous and mesoporous $\mathrm{SiO}_{2}$ : effect of reductive environment, and support structure, Phys. Chem. Chem. Phys. 4 (2002) 827-834. http://dx.doi.org/10.1039/B108545J.

[72] J.J. Rehr, J.J. Kas, F.D. Vila, M.P. Prange, K. Jorissen, Parameter-free calculations of x-ray spectra with FEFF9, Phys. Chem. Chem. Phys. 12 (2010) 5503-5513. http://dx.doi.org/10.1039/B926434E.

[73] A. Haghofer, D. Ferri, K. Föttinger, G. Rupprechter, Who is doing the job? Unraveling the role of $\mathrm{Ga}_{2} \mathrm{O}_{3}$ in methanol steam reforming on $\mathrm{Pd}_{2} \mathrm{Ga} / \mathrm{Ga}_{2} \mathrm{O}_{3}$, ACS Catal. 2 (2012) 2305-2315. http://dx.doi.org/10.1021/cs300480c.

[74] V. Marchionni, M.A. Newton, A. Kambolis, S.K. Matam, A. Weidenkaff, D. Ferri, A modulated excitation ED-EXAFS/DRIFTS study of hydrothermal ageing of $\mathrm{Rh} / \mathrm{Al}_{2} \mathrm{O}_{3}$, Catal. Today 229 (2014) 80-87. http://dx.doi.org/10.1016/j.cattod. 2013.10.082.

[75] R. Kydd, D. Ferri, P. Hug, J. Scott, W.Y. Teoh, R. Amal, Temperature-induced evolution of reaction sites and mechanisms during preferential oxidation of $\mathrm{CO}$, J. Catal. 277 (2011) 64-71. http://dx.doi.org/10.1016/j.jcat.2010.10.009.

[76] D. Ferri, M.S. Kumar, R. Wirz, A. Eyssler, O. Korsak, P. Hug, A. Weidenkaff, M.A. Newton, First steps in combining modulation excitation spectroscopy with synchronous dispersive EXAFS/DRIFTS/mass spectrometry for in situ time resolved study of heterogeneous catalysts, Phys. Chem. Chem. Phys 12 (2010) 5634-5646. http://dx.doi.org/10.1039/B926886C.

[77] C.F.J. König, J.A. van Bokhoven, T.J. Schildhauer, M. Nachtegaal, Quantitative analysis of modulated excitation X-ray absorption spectra: enhanced precision of EXAFS fitting, J. Phys. Chem. C 116 (2012) 19857-19866. http://dx.doi.org/ $10.1021 /$ jp306022k.

[78] G.L. Chiarello, D. Ferri, Modulated excitation extended X-ray absorption fine structure spectroscopy, Phys. Chem. Chem. Phys 17 (2015) 10579-10591. http://dx.doi.org/10.1039/C5CP00609K.

[79] D. Ferri, M.A. Newton, M. Di Michiel, G.L. Chiarello, S. Yoon, Y. Lu, J. Andrieux, Revealing the dynamic structure of complex solid catalysts using modulated excitation X-ray diffraction, Angew. Chem. Int. Ed. 53 (2014) 8890-8894. http:// dx.doi.org/10.1002/anie.201403094.

[80] D. Ferri, M.A. Newton, M. Di Michiel, S. Yoon, G.L. Chiarello, V. Marchionni, S.K. Matam, M.H. Aguirre, A. Weidenkaff, F. Wen, J. Gieshoff, Synchrotron high energy X-ray methods coupled to phase sensitive analysis to characterize aging of solid catalysts with enhanced sensitivity, Phys. Chem. Chem. Phys. 15 (2013) 8629-8639. http://dx.doi.org/10.1039/C3CP44638G.

[81] J. Szlachetko, J. Sá, M. Nachtegaal, U. Hartfelder, J.C.l. Dousse, J. Hoszowska, D.L.A. Fernandes, H. Shi, C. Stampfl, Real time determination of the electronic structure of unstable reaction intermediates during $\mathrm{Au}_{2} \mathrm{O}_{3}$ reduction, Phys. Chem. Lett. 5 (2014) http://dx.doi.org/10.1021/jz402309s.

[82] V. Bushuev, L. Samoylova, H. Sinn, T. Tschentscher, Temporal and coherence properties of hard x-ray FEL radiation following Bragg diffraction by crystals, in: M.S. del Rio (Ed.), Proc. SPIE 8141, Advances in Computational Methods for X-Ray Optics II, 2011, pp. 81410T. http://dx.doi.org/10.1117/12.893054.

[83] J. Szlachetko, M. Nachtegaal, E. de Boni, M. Willimann, O. Safonova, J. Sá, G. Smolentsev, M. Szlachetko, J.A. van Bokhoven, J.C.l. Dousse, J. Hoszowska, Y. Kayser, P. Jagodzinski, A. Bergamaschi, B. Schmitt, C. David, A. Lücke, A von Hamos X-ray spectrometer based on a segmented-type diffraction crystal for single-shot $\mathrm{x}$-ray emission spectroscopy and time-resolved resonant inelastic x-ray scattering studies, Rev. Sci. Instrum 83 (2012) 103105. http://dx.doi.org/ $10.1063 / 1.4756691$.

[84] G. Geloni, V. Kocharyan, E. Saldin, A novel self-seeding scheme for hard X-ray FELs, J. Mod. Opt. 58 (2011) 1391. http://dx.doi.org/10.1080/09500340.2011. 586473. 